\title{
Using BMI to Determine Cardiovascular Risk in Childhood: How Do the BMI Cutoffs Fare?
}

\author{
Asheley Cockrell Skinner, PhDa,b, Michelle L. Mayer, PhD, MPH ${ }^{a}, \mathrm{C}$, Kori Flower, MD, MS, \\ MPH $^{d}$, Eliana M. Perrin, MD, MPHe ${ }^{\text {, and Morris Weinberger, PhD }}{ }^{a}, f^{\prime}$ \\ aDepartment of Health Policy and Management, University of North Carolina at Chapel Hill, \\ Chapel Hill, North Carolina \\ bSchool of Nursing, University of North Carolina at Chapel Hill, Chapel Hill, North Carolina \\ ${ }^{\circ}$ Cecil G. Sheps Center for Health Services Research, University of North Carolina at Chapel Hill, \\ Chapel Hill, North Carolina \\ ${ }^{d}$ Charles Drew Community Health Center, Piedmont Health Services, Burlington, North Carolina \\ eDivision of General Pediatrics and Adolescent Medicine, Department of Pediatrics, School of \\ Medicine, University of North Carolina at Chapel Hill, Chapel Hill, North Carolina \\ ${ }^{f}$ Center for Health Services Research in Primary Care, Durham Veterans Affairs Medical Center, \\ Durham, North Carolina
}

\section{Abstract}

OBJECTIVE-Although adverse health outcomes are increased among children with BMI above the 85th (overweight) and 95th (obese) percentiles, previous studies have not clearly defined the BMI percentile at which adverse health outcomes begin to increase. We examined whether the existing BMI percentile cutoffs are optimal for defining increased risk for dyslipidemia, dysglycemia, and hypertension.

METHODS-This was a cross-sectional analysis of the National Health and Nutrition Examination Survey from 2001 to 2006. Studied were 8216 children aged 6 to 17 years, representative of the US population. BMI was calculated by using measured height and weight and converted to percentiles for age in months and gender. Outcome measures (dyslipidemia, dysglycemia, and hypertension) were based on laboratory and physical examination results; these were analyzed as both continuous and categorical outcomes.

RESULTS-Significant increases for total cholesterol values and prevalence of abnormal cholesterol begin at the 80th percentile. Significant increases in glycohemoglobin values and prevalence of abnormal values begin at the 99th percentile. Consistent significant increases in the prevalence of high or borderline systolic blood pressure begin at the 90th percentile.

CONCLUSIONS-Intervening for overweight children and their health requires clinical interventions that target the right children. On the basis of our data, a judicious approach to screening could include consideration of lipid screening for children beginning at the 80th percentile but for dysglycemia at the 99th percentile. Current definitions of overweight and obese may be more useful for general recognition of potential health problems and discussions with parents and children about the need to address childhood obesity.

Copyright $\odot 2009$ by the American Academy of Pediatrics

Address correspondence to Asheley Cockrell Skinner, PhD, CB 7411, Chapel Hill, NC 27599. asheley@ unc.edu.

FINANCIAL DISCLOSURE: The authors have indicated they have no financial relationships relevant to this article to disclose. 
WHAT'S KNOWN ON THIS SUBJECT: Previous research has shown that cardiovascular risk factors are related to the currently used definitions of obesity in children but has not specified the BMI percentiles at which risk increases.

WHAT THIS STUDY ADDS: Nationally representative data indicate greater risk for abnormal lipid values in children who are not considered overweight by current definitions, risk for diabetes only in very obese children, and risk for hypertension at the 90th percentile of BMI.

\section{Keywords}

obesity; overweight; cardiovascular risk; children

Addressing the "epidemic" of childhood obesity clinically requires a definition of obese that is readily determined and accepted by both physicians and patients. Excess adipose tissue is the basis for defining obesity scientifically, but the general public typically assumes that "overweight" and "obese" are related to adverse health outcomes. BMI percentiles correlate well with measures of adiposity, and specific cutoffs are used to define overweight (from the 85 th to $<95$ th percentiles) and obesity ( $\geq 95$ th percentile) for children. ${ }^{1,2}$ These percentiles were used because they track well to the now commonly used BMI definitions for adults (over-weight: BMI $\geq 25 \mathrm{~kg} / \mathrm{m}^{2}$; obese: $\mathrm{BMI} \geq 30 \mathrm{~kg} / \mathrm{m}^{2}$ ). These adult definitions, however, are not without their own controversy and questions in terms of their relationship to health. ${ }^{3}$ Although many studies have shown that these cutoffs are related to multiple measures of health, no systematic relationships to current health outcomes formed the basis of the definitions. ${ }^{4}$ In fact, some studies have found that these categories do not have the substantial relationship to risk factors and health outcomes that would justify intense efforts for individuals to improve their weight and, in fact, suggest that greater weight is protective in certain health outcomes. ${ }^{5-7}$ These previous studies raise the following question: Are the BMI percentile cutoff points that are used to define overweight and obesity the best indicators of undesirable health outcomes for children and adolescents?

The 85th and 95th percentiles seem to be the points at which older adolescents stabilize to BMI values near the $25-\mathrm{kg} / \mathrm{m}^{2}$ and $30-\mathrm{kg} / \mathrm{m}^{2}$ cutoffs used for adults. ${ }^{8}$ By using these percentile cutoffs, overweight and obesity in childhood clearly predict overweight and obesity in adulthood, ${ }^{9}$ which alone may be an argument to use them. Many previous studies have also found that children who are overweight or obese as defined by current BMI percentile cutoffs are at increased risk for a multitude of health conditions. ${ }^{10-13}$ Although it is clear from this body of work that adverse health outcomes are increased once children are above the 85th percentile (overweight) and 95th percentile (obese), previous studies have not clearly defined the BMI percentile at which adverse health outcomes begin to increase. We were interested in examining whether the existing BMI percentile cutoffs are optimal for defining increased risk for specific adverse health outcomes in childhood. Dyslipidemia, dysglycemia, and hypertension are adverse health outcomes that are associated with overweight ${ }^{13}$ and are especially important because they increase risk for cardiovascular disease. We used a nationally representative sample of children to examine the relationship of BMI to these adverse health outcomes in childhood.

\section{METHODS}

The National Health and Nutrition Examination Survey (NHANES) is a stratified, multistage probability sample of the civilian, noninstitutionalized population of the United States. ${ }^{14}$ It comprises 2 major components, both of which were used in this study. The first is a household interview conducted with a proxy (typically a parent) for children who are 
younger than 16 and with minors themselves if they are $\geq 16$ and the parent or guardian consents. The second component is a health examination, performed in a mobile examination center, which includes a physical examination and laboratory testing of urine and blood specimens.

Our sample includes 8216 children in the United States who were aged 6 to 17 years, the ages at which childhood obesity is most prevalent, and participated in 1 of the NHANES assessments from 2001 to 2006. Underweight children ( $<5$ th percentile for BMI) were excluded because they are more likely to have health conditions related to being underweight, thereby not contributing to a clear picture of the differences between healthyweight and overweight children.

BMI (weight in kilograms divided by height in meters squared) was calculated by using measured height and weight from the health examination and converted to percentiles for age in months and gender by using the Centers for Disease Control and Prevention's SAS programming code developed for that purpose. ${ }^{15} \mathrm{We}$ used current recommendations to define children as very obese (BMI $>99$ th percentile), obese (BMI $>95$ th percentile and $<99$ th percentile), and over-weight (BMI > 85th percentile and <95th percentile) for age and gender. ${ }^{2}$ The cutoffs for these percentiles were developed by using data from the original National Health Examination Survey and later NHANES from 1963 to 199416; therefore, the percentiles do not reflect the distribution of the current US population but are historical measures applied to the current population.

Outcome measures (dyslipidemia, dysglycemia, and hypertension) were based on laboratory and physical examination results. Definitions of normal, borderline, and abnormal lipid measurements come from the American Academy of Pediatrics (AAP) ${ }^{17,18}$ and the American Heart Association standards (Appendix). ${ }^{19}$ The primary diabetes-related measure is fasting plasma glucose, which uses the definition from the American Diabetes Association..$^{20}$ Total cholesterol and high-density lipoprotein (HDL) cholesterol are not fasting measures, whereas triglycerides and low-density lipoprotein (LDL) are measured after a minimum of 8.5 hours of fasting. Because fasting plasma glucose levels were available only for a subsample of the population, glycohemoglobin was also used. ${ }^{20,21}$ Hypertension was defined by using percentiles of systolic and diastolic values on the basis of height percentile, age, and gender. Values $>95$ th percentile were regarded as hypertensive, and values between the 90th and 95th percentiles were considered borderline. ${ }^{22} \mathrm{We}$ also used data on age (at examination when height and weight were measured), socioeconomic status (defined as poor when the family's income is $<100 \%$ of the federal poverty level [FPL]), insurance status (ever uninsured in previous year, private insurance, or public insurance), and race (Hispanic, non-Hispanic black, non-Hispanic white, or other).

BMI percentiles were divided into 5-point increments (eg, 5-9, $10-14)$. The only exception was that children at $\geq 99$ th percentile composed a single category. Outcome measures (dyslipidemia, dysglycemia, and hypertension) were analyzed both as continuous and categorical outcomes. For the former, we examined mean laboratory/clinical values, correcting for survey design. Each point was compared by using a $t$ test to determine whether the mean value of each cardiovascular risk factor at each BMI percentile increment was significantly different from the mean value of all lower BMI percentile increments combined.

We used a similar approach for categorical outcomes (the prevalence of a specific condition). Again, $t$ tests were used to determine whether the prevalence rate of abnormal values of each cardiovascular risk factor at each BMI percentile increment was significantly different from the prevalence rate of all lower BMI percentile increments combined. 
Finally, we used logistic regression to examine unadjusted and adjusted odds ratios for each health outcome, comparing each 5-point BMI percentile increment from the 80th percentile and above. We chose to analyze the 80th percentile and above because our analyses indicated an apparent increase in cardiovascular risk factors at the 80th percentile. All BMI values from the 5th to 79th percentiles were used as the referent group. Odds ratios are presented as unadjusted and adjusted for age, gender, FPL, race and ethnicity, and insurance status.

Correction for the complex survey design was done by using the information on sampling methods and the weights included with each data set. This included applying probability weights to make estimates representative of the US population, as well as adjusting for the stratification and sampling units of the survey design to develop correct estimates of the variances. This was done by using the svy commands in Stata 10.0 (College Station, TX) for all analyses.

\section{RESULTS}

Using the measured values of height and weight in NHANES, $65.6 \%$ of children were healthy weight, $16.7 \%$ were overweight, and $17.7 \%$ were obese. The average age was 11.6 years, with equal numbers of girls and boys. The race distribution was $60.5 \%$ white, $15.0 \%$ black, $17.9 \%$ Hispanic, and 6.6\% other race. Most children lived above the FPL, although $20.4 \%$ lived below the FPL, as defined by the poverty-income ratio. Most children were insured, either through private insurance $(52.0 \%)$ or public coverage $(23.4 \%) ; 24.6 \%$ were uninsured at some point in the previous year. There were no signifi-cant differences in weight category by age or gender. Overweight was more prevalent among black and Hispanic children and among children who were uninsured or living in poverty.

Although the subsections that follow give detailed results for each type of risk factor studied, Table 1 presents mean values for each cardiovascular risk factor at each BMI percentile grouping. In general, a pattern emerged whereby laboratory values for lipid values began to rise at $\sim 80$ th percentile and at the 99 th percentile for glycemic values, although the mean values were still in the normal range. Although there were some differences in effect sizes when boys and girls were analyzed separately (data not shown), there were very limited differences in the weights at which statistically significant increases were seen. We present only the combined analyses to represent better the current clinical use of BMI percentiles to define obese and overweight, which do not differ by gender.

\section{Dyslipidemia}

Examining mean values at all BMI values showed higher values for total cholesterol and LDL cholesterol beginning at the 80th percentile (Table 2). HDL showed a more linear relationship to BMI, with significant decreases in HDL value beginning at the 60th percentile and continuing through the 99th percentile. Significant increases in triglycerides were not noted until the 90th percentile. Controlling for age, race, ethnicity, income, and insurance status, the risk for high or borderline total cholesterol was greater at the 80th percentile, low HDL at the 85th percentile, and high LDL and high triglycerides at the 90th percentile (Fig 1).

\section{Dysglycemia}

Significantly higher values for fasting plasma glucose and glycohemoglobin were seen at the 99th percentile (Table 1). Controlling for age, race, ethnicity, income, and insurance status, the prevalence of abnormal glycohemoglobin and glucose was greater at the 99th percentile (Fig 2). 


\section{Hypertension}

Controlling for age, race, ethnicity, income, and insurance status, the odds of high or borderline systolic blood pressure increased at the 90th percentile (Fig 3). The odds of high or borderline diastolic pressure increased at the 95 th percentile.

\section{DISCUSSION}

When discussing the "epidemic of childhood obesity," many assume that "obesity" is clearly defined and linearly related to poorer health outcomes during childhood itself. Concern about increases in adiposity in children is particularly warranted when those increases relate to current negative health effects rather than predict adult sequelae. Although a universal BMI percentile cut point is useful for defining obesity, such a measure may be less helpful in clinically identifying children who currently have dyslipidemia, dysglycemia, or hypertension.

Our results suggest that the currently used BMI percentile cutoffs for defining overweight and obesity may not accurately identify children with specific cardiovascular disease risk factors, including dyslipidemia, dysglycemia, and hypertension. On the one hand, our analyses provide evidence that using the 85 th percentile to define overweight may omit a large group of children, those in the $80^{\text {th }}$ to 84 th percentiles, with current cardiovascular disease risk factors including dyslipidemia. Should pediatricians counsel these children as to the risks of their weight, despite having what is defined as "healthy weight?" On the other hand, our results suggest that some risk factors such as dysglycemia are prevalent only at the highest BMI percentiles. The BMI percentile cutoffs for defining overweight/obesity are useful because they provide standard definitions for clinical and research purposes and provide clinicians with guidance about which children are in greatest need of weight management strategies. Our analyses, however, are the first to demonstrate the shortcomings of using a single BMI percentile cut point to determine which children may benefit from screening for health problems that are associated with overweight.

Several guidelines exist to provide clinicians with guidance in determining which children may benefit from screening for cardiovascular disease risk factors such as dyslipidemia, dysglycemia, and hypertension. Recently, the AAP updated its cholesterol screening guideline, pointing to $\mathrm{BMI} \geq 85$ th percentile as a criterion for lipid screening. ${ }^{18}$ Similarly, the American Diabetes Association includes use of the 85th percentile for BMI within its criteria for determining which children need screening for type 2 diabetes. ${ }^{20}$ The AAP Expert Committee on Childhood Obesity also recommends laboratory screening for dyslipidemia and dysglycemia beginning at the 85 th percentile. ${ }^{2}$ In all of these guidelines, the 85th percentile for BMI has been used because it synchronizes well with the definition of overweight and provides clinicians with clear, usable recommendations on laboratory screening. To date, however, there has been a lack of evidence supporting the 85th percentile as the most appropriate cut point for dysglycemia and dyslipidemia screening.

For dyslipidemia, we saw a steep rise in total cholesterol and LDL beginning at the 80th percentile. Our categorical analyses support a similar conclusion: there is a significant increase in prevalence of high or borderline total cholesterol at the 80th percentile. Conversely, for dysglycemia, we observed signifi-cant increases in fasting glucose and glycohemoglobin and overtly abnormal values for these measures only at BMI >99th percentile. Our analyses have implications for current recommendations regarding cardiovascular risk factor screening for overweight children: using overweight (85th percentile) and obesity (95th percentile) as triggers for screening children likely results in missing some children who are technically "healthy weight" but have dyslipidemia and probably subjects many children to glucose screening when they are unlikely to have 
abnormalities. We suggest that although the 85th and 95th percentiles are useful for defining overweight and determining management strategies, clinicians may want to use the exact BMI percentile to determine whether children need dysglycemia and lipid screening. On the basis of our data, a judicious approach to screening could include consideration of lipid screening for children beginning at the 80th percentile but for dysglycemia at the 99th percentile.

Our study is limited first by the fact that we based our risk factor prevalence rates on categories as potentially spurious as BMI cutoffs. The lipid, glycemic control, and hypertension measures used in the study theoretically are related to cardiovascular disease in a continuous manner. Imposing a cut point on biologically continuous measures always carries limitations but is necessary for the practical purpose of clinical decision-making, including determining which children benefit most from screening for dyslipidemia and dysglycemia. For the current analyses, we first examined all cardiovascular risk factors as continuous variables to identify best the BMI percentiles at which substantial risk factors occur. We also analyzed lipid and glycemic control risk factors in categorical form (normal, borderline, abnormal) to allow comparison with other studies that examined the prevalence of dyslipidemia and dysglycemia and to enable us to examine the BMI cut point at which clinically recognized dyslipidemia and dysglycemia occur.

Second, the NHANES measurements may not provide the ideal environment for health assessment. For example, blood pressure fluctuates significantly, and the mobile examination unit may result in invalid assessment of hypertension; however, such differences should not differ by weight status, and NHANES permits analysis of measured health values with a large population.

Third, because NHANES is cross-sectional, we cannot determine the direction of the relationship between obesity and cardiovascular risk factors; however, there is no reason to suspect that cardiovascular risk factors might cause obesity.

Finally, current cardiovascular risk factors are not the only things that should be considered as the danger of childhood obesity. Concerns such as the risk for future obesity and other health problems are also important. Future research should examine the relationship between childhood BMI and other health outcomes, as well as adult health outcomes. Although numerous studies have demonstrated the relationship between childhood obesity and adult obesity, these studies also generally relied on the a priori definitions of obese and overweight.

There may be points of particularly increased risk other than those currently used to define overweight and obese. Such studies would provide even more information for clinicians to use when evaluating and treating obese patients.

\section{CONCLUSIONS}

Intervention for overweight children and their health requires clinical interventions that target the right children. Our findings indicate that cardiovascular risk factor screening efforts should be targeted to the most at-risk children, reaping the largest health benefits, which may involve cholesterol screening of children at the 80th percentile for BMI but diabetes screening only in the very obese category ( $\geq 99$ th percentile). Current definitions of overweight and obese may be more useful for general recognition of potential health problems and discussions with parents and children about the need to address childhood obesity. 


\section{Acknowledgments}

Dr Skinner is supported by National Institute of Nursing Research training grant 5-T32-NR008856. Dr Weinberger has a Senior Research Career Scientist Award from the Health Services Research and Development Service, Department of Veterans Affairs. Dr Perrin is supported by an Eunice Kennedy Shriver National Institute of Child Health and Human Development career development award (5 K23 HD051817).

\section{APPENDIX}

\section{APPENDIX}

Definitions of Abnormal Laboratory and Clinical Values

\begin{tabular}{ll}
\hline Variable & Description \\
\hline Hypertension & Height-, age-, and gender-specific percentiles for blood pressure \\
& High ( $\geq 95$ th percentile) \\
& Borderline ( $\geq 85$ th percentile and $<95$ th percentile $)$ \\
Glycohemoglobin & High $(>5.7 \%)$ \\
& Normal $(<5.7 \%)$ \\
Fasting plasma glucose & Undiagnosed diabetes $(>126 \mathrm{mg} / \mathrm{dL})$ \\
& Impaired fasting glucose $(126-110 \mathrm{mg} / \mathrm{dL})$ \\
& Normal $(<110 \mathrm{mg} / \mathrm{dL})$ \\
Total cholesterol & Acceptable $(<170 \mathrm{mg} / \mathrm{dL})$ \\
& Borderline $(170-200 \mathrm{mg} / \mathrm{dL})$ \\
& High $(>200 \mathrm{mg} / \mathrm{dL})$ \\
HDL cholesterol & Acceptable $(>35 \mathrm{mg} / \mathrm{dL})$ \\
& Low $(<35 \mathrm{mg} / \mathrm{dL})$ \\
LDL cholesterol & Acceptable $(<110 \mathrm{mg} / \mathrm{dL})$ \\
& Borderline $(110-129 \mathrm{mg} / \mathrm{dL})$ \\
& High $(>129 \mathrm{mg} / \mathrm{dL})$ \\
Triglycerides & Acceptable $(<200 \mathrm{mg} / \mathrm{dL})$ \\
& High $(>200 \mathrm{mg} / \mathrm{dL})$ \\
\hline & \\
\hline
\end{tabular}

\section{ABBREVIATIONS}

NHANES National Health and Nutrition Examination Survey

AAP American Academy of Pediatrics

HDL high-density lipoprotein

LDL low-density lipoprotein

FPL federal poverty level

\section{REFERENCES}

1. Dietz WH, Robinson TN. Use of the body mass index (BMI) as a measure of overweight in children and adolescents. J Pediatr 1998;132(2):191-193. [PubMed: 9506622]

2. Barlow SE, Expert Committee. Expert committee recommendations regarding the prevention, assessment, and treatment of child and adolescent overweight andobesity: summary report. Pediatrics 2007;120(suppl 4):S164-S192. [PubMed: 18055651] 
3. Kuczmarski RJ, Flegal KM. Criteria for definition of overweight in transition: background and recommendations for the United States. Am J Clin Nutr 2000;72(5):1074-1081. [PubMed: 11063431]

4. Hubbard VS. Defining overweight and obesity: what are the issues? Am J Clin Nutr 2000;72(5): 1067-1068. [PubMed: 11063427]

5. Oreopoulos A, Padwal R, Kalantar-Zadeh K, Fonarow GC, Norris CM, McAlister FA. Body mass index and mortality in heart failure: a meta-analysis. Am Heart J 2008;156(1):13-22. [PubMed: 18585492]

6. Bozkurt B, Deswal A. Obesity as a prognostic factor in chronic symptomatic heart failure. Am Heart J 2005;150(6):1233-1239. [PubMed: 16338264]

7. Curtis JP, Selter JG, Wang Y, et al. The obesity paradox: body mass index and outcomes in patients with heart failure. Arch Intern Med 2005;165(1):55-61. [PubMed: 15642875]

8. Barlow SE, Dietz WH. Obesity evaluation and treatment: Expert Committee recommendations. Pediatrics 1998;102(3) Available at: www.pediatrics.org/cgi/content/full/102/3/e299.

9. Guo SS, Wu W, Chumlea WC, Roche AF. Predicting overweight and obesity in adulthood from body mass index values in childhood and adolescence. Am J Clin Nutr 2002;76(3):653-658. [PubMed: 12198014]

10. Cook S, Weitzman M, Auinger P, Nguyen M, Dietz WH. Prevalence of a metabolic syndrome phenotype in adolescents: findings from the third National Health and Nutrition Examination Survey, 1988-1994. Arch Pediatr Adolesc Med 2003;157(8):821-827. [PubMed: 12912790]

11. Freedman DS, Dietz WH, Srinivasan SR, Berenson GS. The relation of overweight to cardiovascular risk factors among children and adolescents: the Bogalusa Heart Study. Pediatrics 1999;103(6 pt 1):1175-1182. [PubMed: 10353925]

12. Must A, Strauss RS. Risks and consequences of childhood and adolescent obesity. Int J Obes Relat Metab Disord 1999;23(23 suppl 2):S2-S11. [PubMed: 10340798]

13. Skinner AC, Mayer ML, Flower K, Weinberger M. Health status and health care expenditures in a nationally representative sample: how do overweight and healthy-weight children compare? Pediatrics 2008;121(2) Available at: www.pediatrics.org/cgi/content/full/ 121/2/e269.

14. National Center for Health Statistics. NHANES 2001-2002 General Data Release Documentation. National Center for Health Statistics; Hyattsville, MD: 2005.

15. National Center for Chronic Disease Prevention and Health Promotion, Centers for Disease Control and Prevention. A SAS Program for the CDC Growth Charts. Available at: www.cdc.gov/ nccdphp/dnpa/growthcharts/ resources/sas.htm.

16. Kuczmarski R, Ogden C, Guo S, et al. 2000 CDC growth charts for the United States: methods and development. Vital Health Stat 11 2002;(246):1-190. [PubMed: 12043359]

17. American Academy of Pediatrics. Committee on Nutrition. Cholesterol in childhood. Pediatrics 1998;101(1 pt 1):141-147. [PubMed: 11345978]

18. Daniels SR, Greer FR. Committee on Nutrition. Lipid screening and cardiovascular health in childhood. Pediatrics 2008;122(1):198-208. [PubMed: 18596007]

19. Gidding SS, Dennison BA, Birch LL, et al. Dietary recommendations for children and adolescents: a guide for practitioners - consensus statement from the American Heart Association. Circulation 2005;112(13):2061-2075. [PubMed: 16186441]

20. American Diabetes Association. Type 2 diabetes in children and adolescents. Diabetes Care 2000;23(3):381-389. [PubMed: 10868870]

21. American Diabetes Association. Standards of medical care in diabetes. Diabetes Care 2005;28(suppl 1):S4-S36. [PubMed: 15618112]

22. National High Blood Pressure Education Program Working Group on High Blood Pressure in Children and Adolescents. The fourth report on the diagnosis, evaluation, and treatment of high blood pressure in children and adolescents. Pediatrics 2004;114(2 suppl 4th report):555-576. [PubMed: 15286277] 
A

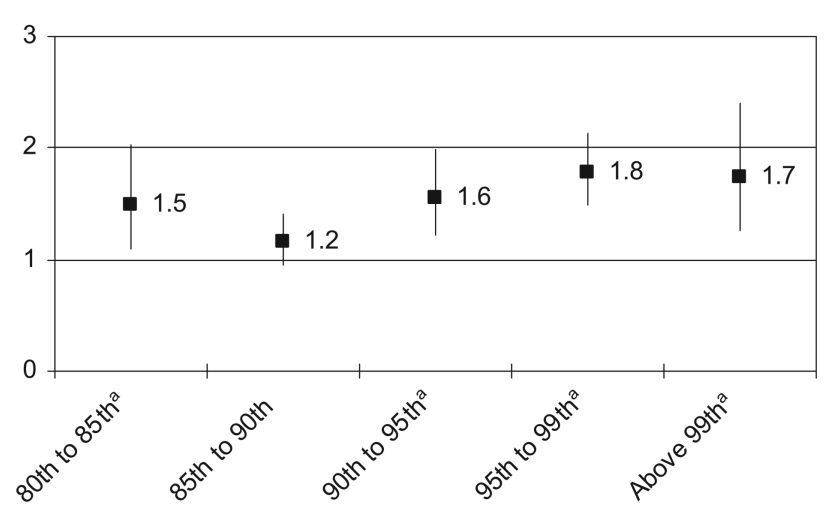

C 6

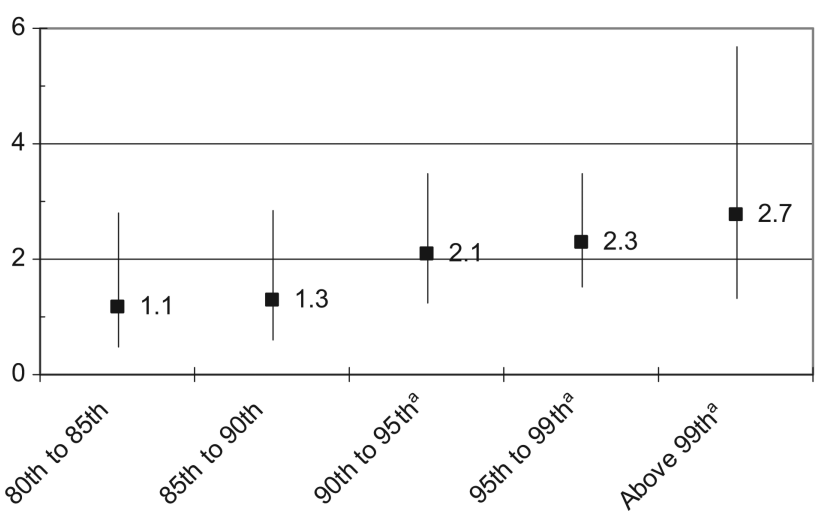

B 25

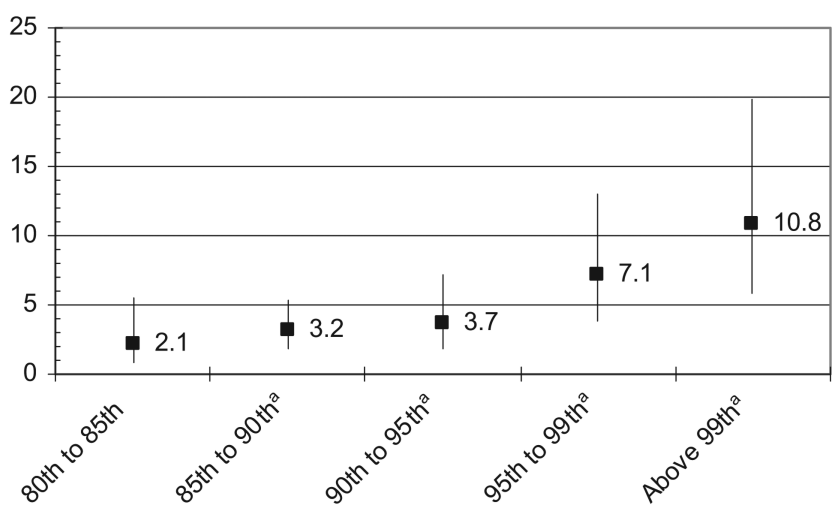

D 100

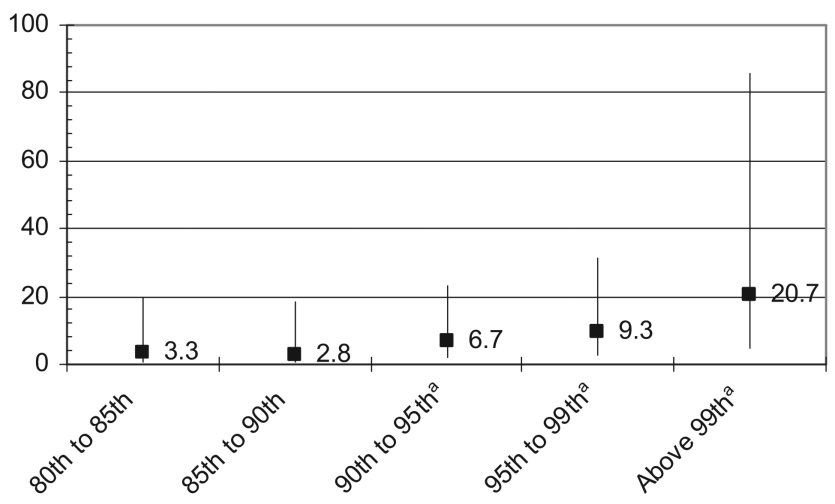

FIGURE 1.

Odds ratios and $95 \%$ confidence intervals from adjusted logistic regression of the effect of weight on likelihood of abnormal lipid values. ${ }^{\mathrm{a}} P<.01$. A, odds of high or borderline total cholesterol; B, odds of low HDL cholesterol; C, odds of high or borderline LDL cholesterol; $\mathrm{D}$, odds of high triglycerides. 
A

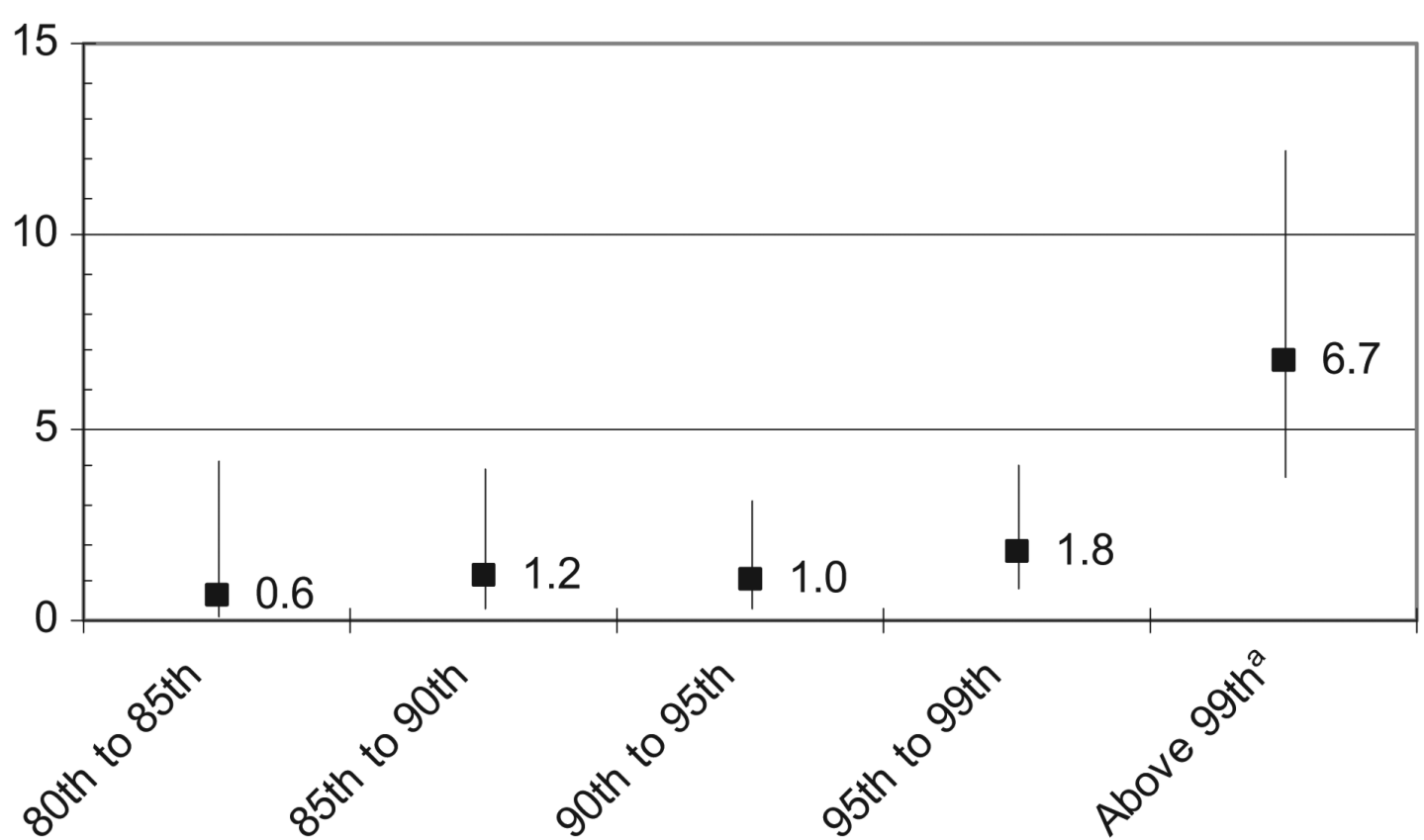

B

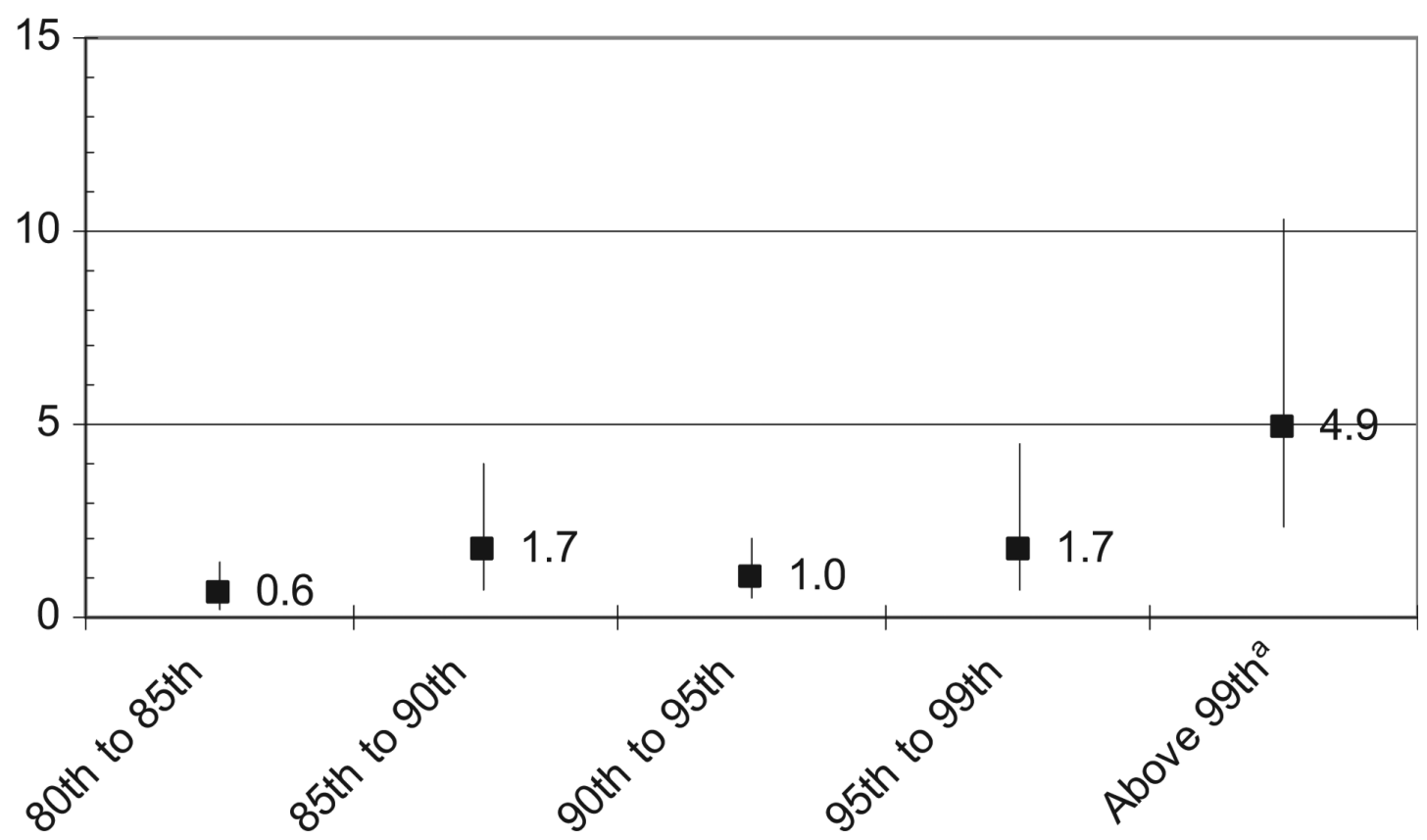

FIGURE 2.

Odds ratios and $95 \%$ confidence intervals from adjusted logistic regression of the effect of weight on likelihood of abnormal glycemic values. ${ }^{\text {a }} P<.01$. A, odds of high glycohemoglobin; B, odds of abnormal glucose. 

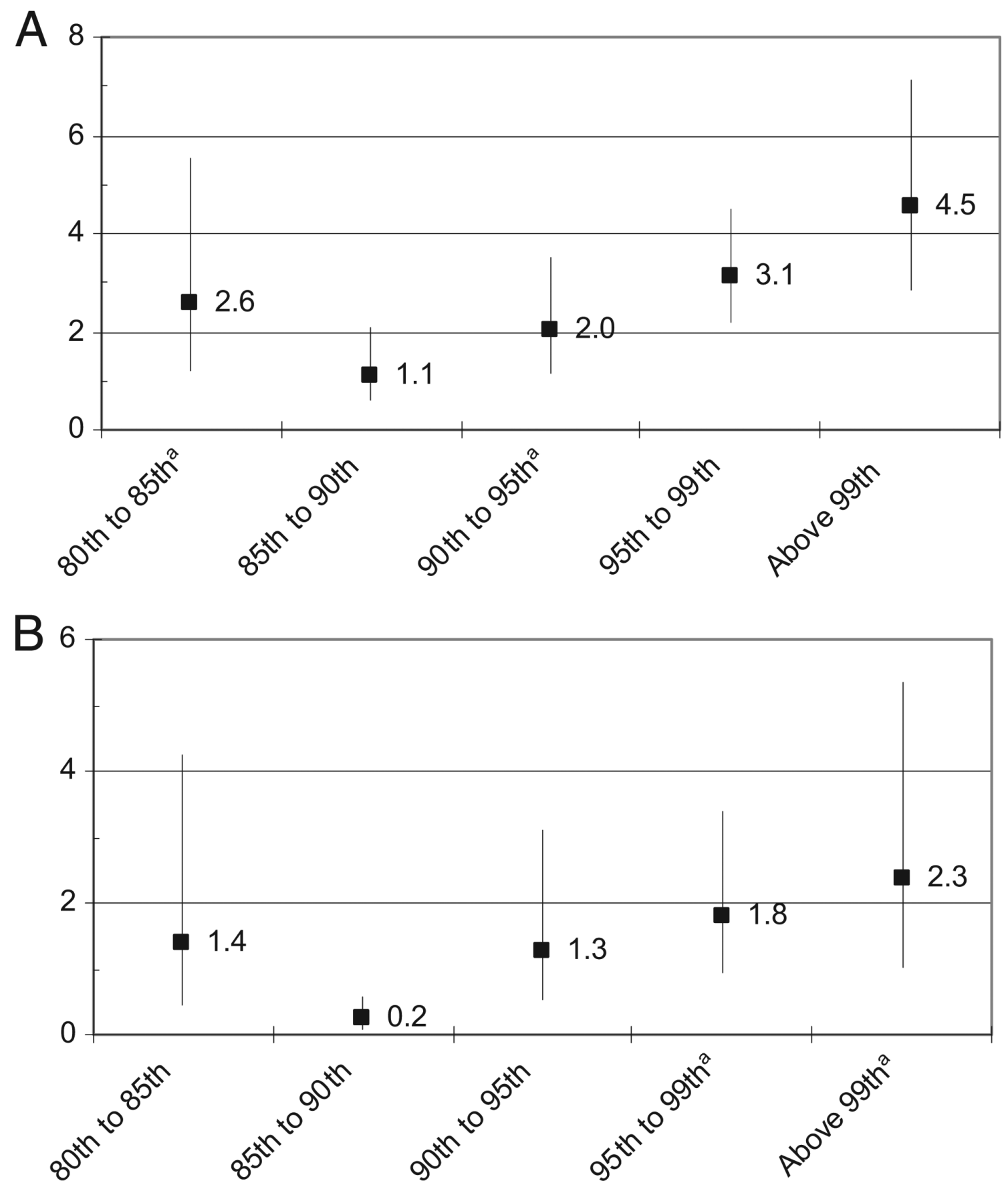

FIGURE 3.

Odds ratios and $95 \%$ confidence intervals from adjusted logistic regression of the effect of weight on likelihood of abnormal blood pressure values. ${ }^{a} P<.01$. A, odds of high or borderline systolic blood pressure; B, odds of high or borderline diastolic blood pressure. 


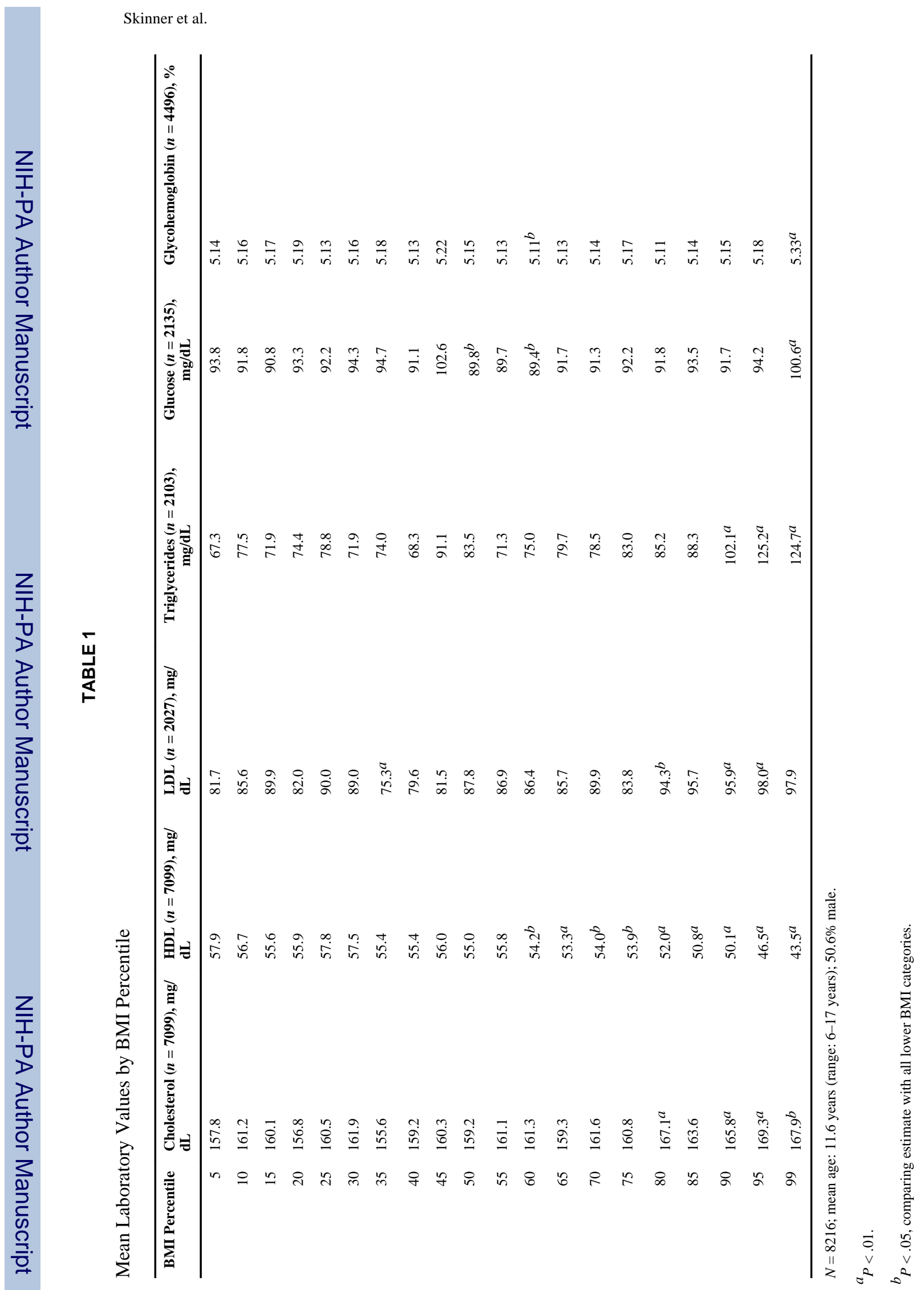

Page 12 


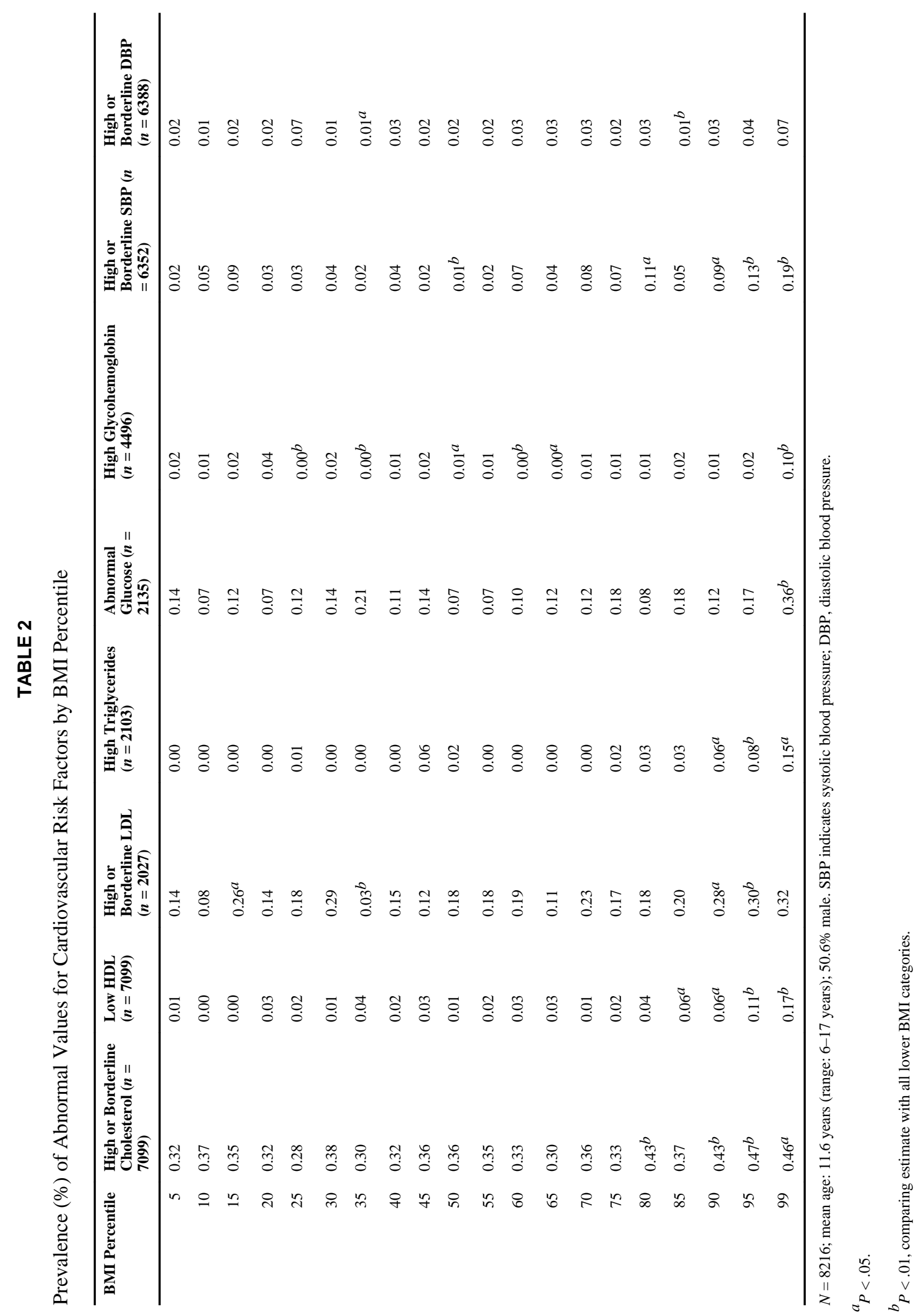

Pediatrics. Author manuscript; available in PMC 2010 July 25. 